\title{
A roadmap for NASA's search for other Earths
}

Charles Beichman, Wesley Traub, Michael Devirian, Adam Burrows

Charles Beichman, Wesley Traub, Michael Devirian, Adam Burrows, "A roadmap for NASA's search for other Earths," Proc. SPIE 5899, UV/Optical/IR Space Telescopes: Innovative Technologies and Concepts II, 589907 (31 August 2005); doi: 10.1117/12.624220 


\title{
A Roadmap for NASA's Search for Other Earths Charles Beichman ${ }^{1, \mathrm{a}, \mathrm{b}}$ Wesley Traub ${ }^{\mathrm{b}}$ and Michael Devirian ${ }^{\mathrm{b}}$ Adam Burrows ${ }^{\mathrm{c}}$ For the NASA Strategic Roadmap (\#4) Team \\ ${ }^{\mathrm{a}}$ Michelson Science Center, M/S 100-22 \\ California Inst. of Technology, Pasadena, CA 91125 \\ ${ }^{\mathrm{b}}$ Jet Propulsion Laboratory, Pasadena, CA 91109 \\ ${ }^{c}$ University of Arizona, Tucson, AZ, 85721
}

\begin{abstract}
The President has identified the search for Earth-like planets around nearby stars as a critical part of NASA's long term Vision for Space Exploration. A suite of space-based missions will determine the incidence of Earth-like planets, detect and characterize the nearest planets, search for signs of life in their atmosphere, as well as make great advances in our understanding of how planetary systems form and evolve. A detailed roadmap lays out the required technology developments, the precursor scientific knowledge, and the capabilities of the relevant missions.
\end{abstract}

Keywords: Earth, planets, telescopes, interferometry, star formation, planetary systems

\section{INTRODUCTION}

"There are innumerable worlds of different sizes. These worlds are at irregular distances, more in one direction and less in another, and some are flourishing, others declining. Some of the worlds have no animal or vegetable life nor any water." Democritus (460 - 370 BCE)

In January 2004 the President articulated a Vision for Space Exploration that explicitly called for "advanced telescope searches for Earth-like planets and habitable environments around other stars" as one of NASA's primary exploration goals. In response to the President's vision NASA undertook to develop a series of strategic roadmaps that included a detailed plan for the search for habitable worlds and life beyond our solar system. Strategic Roadmap \#4 calls for space observatories of dramatic scope to find planets the size of the Earth orbiting nearby stars within the star's habitable zone, characterize the atmospheres of those planets in a search for signatures of habitability or even of life itself, improve our understanding of the formation of planetary systems, and follow the history of elements needed for life from their formation in the first generation of stars to their arrival onto nascent terrestrial planets via cometary impacts. At least five space missions will be required to complete these goals: the Kepler satellite to determine the frequency of Earth-sized planets using transits of planets in front of 100,000 distant sun-like stars; the Space
Table 1. Roadmap Team Members

Adam Burrows, University of Arizona, co-chair

David Spergel, Princeton University, co-chair

Ghassem Asrar, Science Mission Directorate, co-chair

Jerry Chodil, Ball Aerospace (retired)

Tom Greene, Ames Research Center

Maureen Heath, Northrop Grumman Space Technology

John Mather, Goddard Space Flight Center

Victoria Meadows, Spitzer Science Center

Geoff Marcy, University of California

Frank Martin, Lockheed Martin (retired)

Neil Tyson, American Museum of Natural History

Alycia Weinberger, Carnegie Institution of Washington

Ex Officio and Liaison

Charles Beichman, Michelson Science Center

Rich Capps, Advanced Planning and Integration Office Coordinator (JPL)

Mike Devirian, Jet Propulsion Laboratory

Edna DeVore, SETI Institute

Anne Kinney, Science Mission Directorate

Eric P. Smith, Mission Directorate Coordinator

${ }^{1}$ chas@pop.jpl.nasa.gov

UV/Optical/IR Space Telescopes: Innovative Technologies and Concepts II, edited by Howard A. MacEwen, Proceedings of SPIE Vol. 5899 (SPIE, Bellingham, WA, 2005) · 0277-786X/05/\$15 · doi: 10.1117/12.624220 
Interferometer Mission (SIM PlanetQuest) to survey nearby stars looking for objects of a few Earth masses and to measure their masses and orbital parameters; the James Webb Space Telescope (JWST) to observe the earliest galaxies to understand better the formation of the elements as well as to study planet formation in the disks of young stars; the Terrestrial Planet Finder (TPF) missions, including a visible coronagraph $(T P F-C)$ and an infrared interferometer $(T P F-$ I), to provide physical characterization of planets including spectroscopic measurement of planetary atmospheres and a search for the signposts of life.

These missions will take at least a generation to complete and will probably follow a path different from today's roadmap, but by the end of this program we will have addressed the question of "Other Worlds" that dates back almost 2,500 years with the scientific tools of the $21^{\text {st }}$ century. Whether we find life on neighboring planets or not, we will have deepened our understanding of our cosmic origins and come closer to determining whether or not we are alone in the universe. This article provides a summary of and excerpts from the overall roadmap as developed by the team listed in Table 1. The entire roadmap can be found at: http://planetquest.jpl.nasa.gov/documents/SearchforEarth-LikeCDB59.pdf

\section{THE SCIENTIFIC CHALLENGES}

The roadmap has two scientific themes that will lead to these and other discoveries: the search for extrasolar planets and their direct detection and characterization; and the study of the formation and evolution of exoplanetary systems from stellar disks. The roadmap delineates the investigations and the missions that make up these two themes.

\subsection{Do other stars harbor planets like Earth?}

The search for extrasolar planetary systems is well underway, and we now know of over 150 planets outside our solar system, most discovered by NASA-supported ground-based telescopes and with the help of NASA-supported grants. While some of these planets are gas giants similar to Jupiter and Saturn in our Solar System, some of the newly discovered planets have masses as small as 10 times the mass of the Earth. In recent weeks, the first two rocky planets have been identified: one with 7-10 Earth masses orbits so close to its star that is extremely unlikely to be gaseous; the other (HD 149026b) has a mass 60-70 times that of the Earth and, as determined by a combination of transit and radial velocity data, a density 1.4 times that of water.

These recent discoveries have already revealed important insights:

- Planets are quite common. Roughly 7\% of nearby stars harbor a giant planet within 3 AU.

- The number of planets increases as mass decreases towards the mass of an Earth.

- Stars that contain higher abundance of metals are more likely to have planets

- Multiple planets are common, often in resonant orbits

- The number of planets increases with distance from the star.

- Eccentric orbits are common, with only $10 \%$ being nearly circular.

The increasing number of planets with smaller mass suggests that planets with masses below 15 Earth masses, currently undetectable, are even more numerous. Moreover, the correlation with heavy elements supports current planet formation theory that suggests rocky planets would be more numerous than the gas giants. The observations suggest that many nearby stars harbor rocky planets.

Doppler planet search techniques have found all but 5 of the $\sim 150$ known extrasolar planets. Over the next few years, these Doppler planet searches are poised to discover Jupiter-mass planets orbiting at 4-7 AU, providing the first direct comparison of planets in our Solar System to those orbiting at comparable distances from other stars. Jupiter analogs, those in circular orbits having no giant planet inward of them, may be signposts of rocky planets orbiting closer in, thus serving to prioritize target stars for SIM -PlanetQuest, Terrestrial Planet Finder-Coronagraph (TPF-C), and Terrestrial Planet Finder-Interferometer (TPF-I). Doppler work with a precision of $1 \mathrm{~m} / \mathrm{s}$ would allow detection of planets having mass as low as 10 Earth masses, but most easily if they orbit within $0.1 \mathrm{AU}$ of a solar-mass star, a region that is hotter than the corresponding habitable zone. Earth-mass planets orbiting at roughly $1 \mathrm{AU}$ induce a stellar wobble of only 0.1 $\mathrm{m} / \mathrm{s}$, a factor of 10 below the detection threshold of even future Doppler work. Thus, other space-borne techniques are needed to find Earth-like planets, first indirectly with SIM and then directly with TPF-C and TPF-I. 
While current observations suggest that rocky planets may be common, their abundance is quite uncertain. Kepler will address this question statistically by surveying stars 200-600 parsecs away (one parsec is equal to 3.26 light-years). Kepler will detect Earth-sized planets (and larger) through their rare alignment with the host star, dimming it by one part in 10,000. Planets found by Kepler will be too distant for follow-up by SIM-PlanetQuest or TPF. SIM-PlanetQuest and TPF will survey nearby stars and determine the abundance of nearby Earth-like planets.

\subsection{What are the properties of these planets?}

Discovery will be but the first step in our exploration of extrasolar rocky planets. Next, we will want to learn the basic properties of each newly discovered planet. The diversity of rocky worlds is likely much greater than that represented by Mercury, Venus, Earth, and Mars. SIM-PlanetQuest, TPF-C and TPF-I will begin the process of exploring these new planets by measuring their fundamental properties:

- Mass. The SIM-PlanetQuest mission will directly measure the masses of the larger rocky planets. The mass of a rocky planet determines whether it can retain molecules in its atmosphere. The presence of greenhouse gases in the atmosphere determines the planet's temperature. Mass also sets the geochemical and thermal structure of the interior of the planet, which dictates the presence of plate tectonics (affecting the cycling of surface material), active volcanism, and magnetic dynamos (that provide magnetic protection from cosmic rays). Mass discriminates ice-giants from rocky planets that otherwise differ little in radius.

- Surface Temperature and Radius. The temperature of rocky planets will be measured unambiguously by the combination of TPF-C and TPF-I. The direct images themselves provide orbital distances, which imply temperatures from radiative equilibrium, albeit with an uncertainty due to the unknown albedo (light reflection fraction) and greenhouse effects. By combining measurements of the reflected visible fluxes (setting albedo) made by TPF-C and of the mid-IR fluxes (setting planet luminosity) made by TPF-I, we can uniquely determine

\begin{tabular}{|c|c|c|c|c|}
\hline \multicolumn{2}{|r|}{ Parameters } & SIM & TPF-C & TPF-I \\
\hline \multicolumn{5}{|c|}{ Orbital Parameters } \\
\hline- & Stable orbit in habitable zone & $\checkmark$ & & \\
\hline \multicolumn{5}{|c|}{ Characteristics for habitability } \\
\hline- & Temperature & & & $\checkmark$ \\
\hline- & Temperature Variability due to distance changes & $\checkmark$ & & \\
\hline- & Radius & & + & + \\
\hline- & Albedo & & + & + \\
\hline- & Mass & $\checkmark$ & & \\
\hline- & Surface gravity & + & + & + \\
\hline- & Atmospheric and Surface Composition & & $\checkmark$ & $\checkmark$ \\
\hline- & Atmospheric conditions & & $\checkmark$ & $\checkmark$ \\
\hline- & Presence of water & & $\checkmark$ & $\checkmark$ \\
\hline- & Temporal Variability of composition & & $\checkmark$ & $\checkmark$ \\
\hline \multicolumn{5}{|c|}{ Solar System Characteristics } \\
\hline- & Influence of other planets & $\checkmark$ & & \\
\hline- & Presence of comets or asteroids & & $\checkmark$ & \\
\hline \multicolumn{5}{|c|}{ Indicators of Life } \\
\hline- & Atmospheric Biosignatures (e.g., $\mathrm{O}_{2}, \mathrm{O}_{3}, \mathrm{CH}_{4}$ ) & & + & + \\
\hline- & Surface Biosignatures (e.g., vegetation red edge) & & $\checkmark$ & \\
\hline
\end{tabular}

Table 2. Physical Parameters Determined by SIM, TPF-C, and TPF-I. Red plus signs mean that all the missions are reauired to determine the parameter: black checks mean that the indicated parameter can. in princinle. be obtained bv 
the radius and surface temperature of the planets. These measurements by TPF-C and TPF-I will establish the habitability of each detected rocky planet. Table II-1 summarizes the scientific synergies between SIM, TPF-C, and TPF-I.

- Atmospheric Composition. TPF-C and TPF-I will acquire low-resolution spectra of rocky planets enabling the first measures of the chemical composition of their atmospheres. The spectroscopic observations will be designed to detect oxygen, ozone, carbon dioxide, and methane in the planet's atmosphere. These spectroscopic observations will also be essential for our search for biomarkers (next section).

- $\quad$ Surface Properties. TPF-C/TPF-I will search for temporal variability in the brightness of the rocky planets caused by the rotation of surface features and clouds. TPF-C can get direct spectral measurements of planetary surface composition (rock, ocean, ice, vegetation) and TPF-I will be able to discriminate $\mathrm{CO}_{2}$ ice-covered worlds with thin atmospheres. By measuring such variations over many rotation periods, these observations will reveal whether the planet has clouds, oceans, and continents. These temporal observations will also reveal the rotation period of the planet and could detect annual global variations in planetary properties. Remote observations of the Earth would reveal significant daily variations in its brightness.

By measuring these basic properties, the planned suite of missions will determine whether any of the nearby planets are suitable environments for detectable life.

\subsection{How will we detect the presence of life?}

Our search plans assume that the effects on a planet of even the most basic forms of life are global, and that biosignatures from the planet's atmosphere or surface will be recognizable in the disk-averaged spectrum of the planet. Observations and exploration of our own and other planets in our Solar System, and ongoing astrobiology research, have taught us that signs of life can only be conclusively recognized in the context of the overall planetary environment.

The Earth has known surface biosignatures from vegetation, and several atmospheric biosignatures, including the characteristic spectra of life-related compounds like oxygen - produced by photosynthetic bacteria and plants - and its photochemical product, ozone. A more robust atmospheric biosignature is the simultaneous presence of oxygen or ozone and a reduced gas, such as methane $\left(\mathrm{CH}_{4}\right)$ or nitrous oxide $\left(\mathrm{N}_{2} \mathrm{O}\right)$, which are also produced by life. Although these latter two gases are difficult to detect in the Earth's current atmosphere they and other biogenic compounds may be more detectable on Earth-like planets around other stars or during different stages of a planet's history.

Life, and the conditions under which life thrives, may not be identical to those found on Earth. Correspondingly, we must design missions that are as robust as possible in thoroughly characterizing planets of unknown composition, and in searching for the byproducts of metabolisms that may not be familiar to us. To do this, we must observe over the largest wavelength range possible, to provide confirming detections of the same molecular species, and to increase our overall chances of detecting and interpreting biosignatures in the context of their planetary environment. This will maximize the probability of success in our search for extrasolar life and provide crosschecks and verification tests for what could be the most important scientific and cultural discovery of the century. A marginal detection of oxygen in the visible could be verified with a follow-up detection of the stronger ozone in the mid-IR. Having data in two different wavelength regions would also help with the identification of the secondary biomarker gases such as $\mathrm{CH}_{4}$ and $\mathrm{N}_{2} \mathrm{O} . \mathrm{CH}_{4}$ can be observed in both the visible and the thermal infrared, although detection in the mid-IR can be problematic in the presence of strong absorption from water vapor, whereas $\mathrm{N}_{2} \mathrm{O}$ can only be seen in the mid-IR. In addition, many metabolic byproducts of life have absorption features that are accessible only in the mid-IR. On the other hand, surface signatures of life, such as those from leafy plants on Earth, can only be detected in the visible range. Thus, robust detection and quantification of biosignatures from the widest possible range of potential metabolisms would require data in both wavelength regions.

\subsection{How does star formation lead to planet formation?}

Astronomers have developed a star formation scenario based on two decades of observation, computer simulation, and theoretical calculation. After a dramatic initial collapse, a protostar grows for a few hundred thousand years as gas and dust flow onto it from the surrounding cloud. A swirling flattened disk of gas and dust forms, through which additional 
mass flows onto the young stellar object. Eventually the new star stabilizes, the fusion of elements in its core producing energy that counteracts the compression of gravity. In broad outline, observations from the ground and from space (e.g., using the Hubble Space Telescope [HST] and Spitzer) have verified this scenario. However, significant questions remain within this paradigm. What triggers the cloud to collapse at all? What sets the mass of the final star? How does the presence of neighboring protostars affect the material left in the disk? For how long does the disk retain material that might form a planetary system? What determines if the surrounding material forms a planetary system, a second star, or merely escapes back into empty space?

\subsubsection{How do stars and disks interact?}

If star-forming cores did not spin as they collapsed, there would be no disks in which to form stars or planets. Yet, if the rotation rates were too great the disks would spin apart. Is rotation controlled by a universal process that makes all disks hospitable places for planet formation? Once the disks form, they influence the ultimate characteristics of stars, such as mass and rotation speed, while the stars drive mixing and chemical processes in the disks. X-rays and ultraviolet photons generated as stars pull in gas from the disk return to the disk and ionize its upper layers, feeding the star even faster and producing complex chemical reactions in the disk. Simultaneously, energetic photons from neighboring stars may be penetrating the disk, so the final planetary system may depend on stellar environment. The Keck Interferometer, Stratospheric Observatory for Infrared Astronomy (SOFIA), Herschel, the James Webb Space Telescope (JWST), the Single-Aperture Far-InfraRed mission (SAFIR), and a future Large UV/Optical mission will address how the presence of terrestrial and giant planets is related to disk dynamics, stellar mass, age, and magnetic activity, stellar binarity, and/or the presence of surrounding stars in a cluster.

\subsubsection{Where do planets form?}

Knowledge of planetary system architectures, that is, the nature and position of all component planets, and the presence of comets and asteroids, is important for understanding the likelihood of habitable planets. Giant planets, which are unlikely to directly harbor life, dynamically constrain the orbits available for terrestrial planets. Giant planets are the older siblings both the bullies and protectors of the terrestrial planets. How they stir up the disk determines how many comets and asteroids survive to bombard smaller worlds with either sterilizing intensity or with life-bringing chemicals. Giant planets in eccentric orbits are less likely to allow terrestrial planets to orbit stably in the habitable zone. However, gas giants might be necessary for shielding terrestrials from life-damaging impacts of comets.

One of the exciting and unexpected results of the planet searches to date is the large number of systems unlike our own. Gas giant planets have been found to orbit closer to their stars than Mercury about the Sun. Among the unsolved problems in planet formation today is not just how to form giants, but how to move them to their present locations. Some appear to have migrated large distances while others, such as Jupiter, appear to have mainly remained in place. Many gas giants also have substantial eccentricities - coming closer and further from their stars over their orbits. However, these systems exist only for a minority (10-20\%) of stars, leaving open the possibility that many stars have planetary systems more like our own. Only with missions such as SIM will we obtain an unbiased view of planetary architectures.

\subsection{How do the components of life come to reside on terrestrial planets?}

Life, as we know it, depends entirely on the complex chemistry of compounds built around carbon atoms, known as organic compounds. We live on the rocky Earth with abundant silicates, iron-bearing minerals, and surface water. We now know that the Universe was not born with these materials, but that the stars themselves are the sites of their manufacture. This discovery that the heavy elements essential for life come directly from stars demonstrates the importance of understanding the creation and distribution of heavy elements and organic molecules throughout the Universe.

\subsubsection{When did the Universe form the raw materials necessary for planets and life?}

The buildup of these heavy elements did not happen all at once. We have learned how these elements are made in stars and how they can be recycled into future generations of stars and potential planetary systems. At the ends of their lives massive stars explode and less massive stars slowly shed gas enriched with these heavy elements. In each cycle the abundance of heavy elements increases as the "ash" of nuclear burning in the centers of stars is added to the mix. We can roughly chart the increase in heavy elements over the generations of stars born over the 12 billion year lifetime of our Milky Way Galaxy and compare it with similar processes in other nearby galaxies. With infrared spectroscopic 
observations of very distant galaxies using JWST, we will soon be able to measure the buildup of elements over cosmic time. In turn, we will better understand the importance of heavy elements for the formation in the Milky Way of planets and, ultimately, life. Such observations will also trace the buildup of gas, solid-state molecules, and dust over cosmic time. This will lead us to understanding the possibility of planets and ultimately life in the Milky Way and the universe as we understand better the importance of heavy elements for the formation of planets. We already know that stars with more heavy elements are more likely to have close-in giant planets. We want to know what metal content is required for various types of planets to form and whether any particular elements are needed to make geologically-active planets such as Earth and the early Mars. JWST and the TPFs will tell us.

\subsubsection{What is the origin of the interstellar medium composition?}

The atoms, dust grains, and hydrocarbon molecules released by dying stars in the Milky Way must follow long circuitous paths in order to be swept up into star forming clouds and eventually processed into materials necessary for life on planets. These newly created materials must spend large amounts of time drifting in the diffuse interstellar medium before they are eventually swept up into dense clouds of gas and dust by gravitational forces. Clouds shield these materials from damaging external radiation, allowing the carbon-nitrogen-oxygen group to form simple volatile molecules such as carbon dioxide, methane, ammonia, and water when they combine with hydrogen atoms (which are all-pervasive) and each other. This combination of volatiles, dust grains, and hydrocarbons is key to forming the chemical building blocks of life in the environments around young stars and their nascent planetary systems. Observations with JWST, SOFIA, and a large UV/optical telescope will all contribute to our understanding of the interstellar medium in our own and distant galaxies.

\subsubsection{How are volatiles and organic molecules delivered to terrestrial planets?}

Physical processes during stellar evolution modify the disk and its constituents. Interstellar dust contains amorphous silicates, but the comets in our Solar System and dust in debris disks around other stars contain a significant fraction of crystalline silicates. These crystals must be formed in hot regions of the disk. Determining where and how these crystalline grains are produced will tell us how the disk is mixed by turbulence, inward accretion, and stellar winds, giving insight to the creation of our own Solar System, as well as others. Near-to-far-infrared spectra with high spatial resolution are necessary to trace the distribution of these important planetary constituents, requiring the Keck and Large Binocular Telescope (LBT) Interferometers, SOFIA, JWST, and SAFIR. As gleaned from the impact record of the early Solar System, while the disks around young stars are clearing, it is likely that these remnant rocky, crystalline asteroids and comets intensely bombard their terrestrial planets. Does this bombardment deliver the carbon-rich material to start the development of life?

\section{RECOMMENDED MISSIONS AND R\&D PROGRAMS}

The technology required to detect and characterize potentially habitable worlds is so challenging that no single mission can provide all the measurements needed. Nor can any one mission be as productive operating alone as it would be working as part of a carefully planned program. Consequently, the search for Earth-like planets is composed of missions that independently take intermediate observational steps, providing valuable scientific results each step along the way. These results, in addition to contributing to the overall scientific body of knowledge, are used to mitigate the risk and uncertainties inherent in the other missions, improving operational efficiencies and measurably increasing the probability of mission success. We discuss briefly the scientific goals and technology challenges of each of the major missions.

\subsection{Space Interferometry Mission (SIM PlanetQuest) and Kepler}

Two missions will carry out a census of planets around solar type stars. Kepler will use the transit technique to search for small decreases in the light output from stars as planets located in edge-on systems move in front of their parent stars. From dips of $1 \%$ from Jupiter-sized planets to dips of $0.01 \%$ from transiting Earths, Kepler will be able to infer the incidence of planets across a large range of sizes and orbital location. These observations will set the scale of the TPF missions by suggesting how large a stellar population (and thus how distant) must be sampled to obtain data on terrestrial planets. Since Kepler will investigate stars that are 500-1,000 pc away, direct detection of light from Kepler's planets will be impossible. However, spectroscopy during transits by JWST will enable a characterization of the atmospheres of some of these planets. 
Kepler will require photometry at one part in 100,000. Since the occultations typically take 4-8 hours, the spacecraft has three multi-hour driving requirements: the pointing jitter must be 100 milliarcseconds or better, the optical system must be sufficiently stable in order to have a stable point spread function, and the CCDs must have stable relative performance.

To search for planets around the nearest stars will require the Space Interferometry Mission (SIM PlanetQuest) which will be capable of detecting Earth-like planets around the closest sun-like stars. SIM will survey the nearest stars and determine gross physical properties of planets such as mass and orbital eccentricity essential to establishing habitability. SIM will add critical information to our growing knowledge about the nearest stars and, thus, help to identify targets most suitable for subsequent observation by Terrestrial Planet Finder (TPF) missions. The knowledge from SIM that particular stars have (or do not have) planets of various masses and orbits, and continued radial-velocity studies, will focus the targeting choices for TPF, thereby increasing the early tempo of direct detection and characterization.

SIM PlanetQuest will find planetary systems by detecting changes to the stars' positions at the micro-arcsecond level due to planetary perturbations. To achieve the necessary astrometric precision requires that two very difficult requirements be met: the first is interferometric picometer metrology needed to make accurate relative measurements of target stars relative to faint reference stars. The second is the end-to-end verification of system performance. Modeling at a new level of sophistication will be needed to verify individual hardware subsystems and then to verify that the subsystems can be tied together to provide the confidence needed before committing to launch. These capabilities must be developed on SIM in order to confidently proceed into TPF-I.

\subsection{James Webb Space Telescope}

JWST will be a multi-purpose observatory equipped with a rich suite of instrumentation along the lines of the Hubble Space Telescope. It will provide imaging, spectroscopy, and basic coronagraphic capabilities at wavelengths from 0.6 to 28 microns to enable observations of everything from solar system objects and planetary disks to the most distant, newly-forming galaxies. JWST requires deployment of precision optical components, and it has benefited from the development of mid-infrared detector arrays and the passive cooling technologies of Spitzer. Key technologies being completed for JWST include the ultra-light deployable segmented beryllium primary mirror, wavefront sensing and control, a multilayer deployable sunshield, radiative cooling to the $30-50 \mathrm{~K}$ level, advanced large format IR detectors of $\mathrm{HgCdTe}$ and Si:As with much lower noise levels than achieved before, micro-shutter arrays for multi-object spectroscopy, and a mechanical cryocooler to cool the mid-IR detectors to $7 \mathrm{~K}$.

\subsection{Terrestrial Planet Finder Missions}

Direct imaging detection and spectroscopic characterization of nearby Earth-like planets will require the Terrestrial Planet Finder (TPF) missions. The first, the TPF Coronagraph (TPF-C), planned for launch in 2014, will suppress the light of the central star to unprecedented levels, allowing it to search for terrestrial planets in $\sim 120$ nearby planetary systems, and thoroughly study 35 . TPF-C will be followed by the TPF Interferometer (TPF-I) about five years later. TPF-I will operate in the mid-IR, will search for terrestrial planets around approximately 500 stars, and will characterize all of those it finds.

Once a terrestrial planet is detected, TPF-C and TPF-I will determine which planets have conditions suitable for life, e.g. a warm, wet atmosphere, and which, if any, show global signs of life, e.g. an oxygen-rich atmosphere due to the effects of photosynthesis. Theoretical, laboratory, and field studies are already under way to learn which "biosignatures" identifiable features in the spectrum of the planet's light — can reveal the presence of life on a distant planet. This research will guide the requirements for the Terrestrial Planet Finders, and help in the design of future telescopes, such as Life Finder. 


\begin{tabular}{|c|c|c|c|}
\hline & Phase 1: 2005-2015 & Phase 2: 2015-2025 & Phase 3: 2025 + \\
\hline $\begin{array}{l}\text { Planet } \\
\text { Detection }\end{array}$ & $\begin{array}{l}\text { a) Measure the frequency of } \\
\text { Earth-like planets in a } \\
\text { statistically representative } \\
\text { sample [COROT, Kepler] } \\
\text { b) Radial velocity surveys detect } \\
\text { additional Jupiter analogs and } \\
\text { nearby planets with less than } \\
10 \mathrm{M}_{\text {Earth }} \text { [Ground] } \\
\text { c) First SIM planet detections }\end{array}$ & $\begin{array}{l}\text { a) Astrometric detection of } \mathrm{M}>3 \\
\mathrm{M}_{\text {Earth }} \text { planets in habitable zone } \\
\text { within } 10 \text { parsecs [SIM] } \\
\text { b) Photometric detection of } \mathrm{M}> \\
0.5 \mathrm{M}_{\text {Earth }} \text { planets in stellar } \\
\text { habitable zone within } 10 \text { parsecs } \\
{[\mathrm{TPF}-\mathrm{C}]} \\
\text { c) Photometric detection of } \mathrm{M}> \\
0.5 \mathrm{M}_{\text {Earth }} \text { planets in stellar } \\
\text { habitable zone within } 100 \\
\text { parsecs [TPF-I] }\end{array}$ & $\begin{array}{l}\text { a) At least an order of } \\
\text { magnitude increase in the } \\
\text { number of directly- } \\
\text { detected terrestrial } \\
\text { planets [LF] } \\
\text { b) Direct detection of } \\
\text { moons in nearby } \\
\text { extrasolar planetary } \\
\text { systems [LF] }\end{array}$ \\
\hline $\begin{array}{l}\text { Planet } \\
\text { Character- } \\
\text { ization }\end{array}$ & $\begin{array}{l}\text { a) Measure atmospheric spectra } \\
\text { of hot Jupiters seen in } \\
\text { transiting events [Ground, } \\
\text { HST, Spitzer, JWST] } \\
\text { b) Measure spectra of brown } \\
\text { dwarfs and giant planets } \\
\text { [JWST] }\end{array}$ & $\begin{array}{l}\text { a) Measure Mass [SIM] } \\
\text { b) Measure radius and surface } \\
\text { temperature [TPF-C+TPF-I] } \\
\text { c) Detect basic atmospheric } \\
\text { composition and presence of } \\
\text { clouds [TPF-C+TPF-I] } \\
\text { d) Determine gross surface } \\
\text { properties [TPF-C, TPF-I] } \\
\text { e) Detect new classes of planets } \\
\text { [SIM, TPF-C, TPF-I] } \\
\text { f) Detect provisional indications of } \\
\text { life [TPF-C, TPF-I] }\end{array}$ & $\begin{array}{l}\text { a) Confirmation of } \\
\text { biomarkers [LF] } \\
\text { b) Search for life on a larger } \\
\text { sample of planets [LF] } \\
\text { c) Search for a variety of } \\
\text { different metabolisms } \\
\text { [LF] } \\
\text { d) Enhance characterization } \\
\text { of planetary systems } \\
\text { [LF] }\end{array}$ \\
\hline $\begin{array}{l}\text { Planet } \\
\text { Formation } \\
\text { and } \\
\text { Habitability }\end{array}$ & $\begin{array}{l}\text { Observe the formation and } \\
\text { evolution of stars, galaxies, and } \\
\text { planetary systems, from the first } \\
\text { luminous objects to debris disks } \\
\text { in our own neighborhood } \\
\text { [Spitzer, SOFIA, Herschel, } \\
\text { JWST] }\end{array}$ & $\begin{array}{l}\text { Observe the development of } \\
\text { conditions for life, from the first } \\
\text { release of the chemical elements in } \\
\text { the first stars, through the } \\
\text { formation of protoplanetary disks, } \\
\text { to the chemistry and physics of the } \\
\text { Solar System [SOFIA, } \\
\text { JWST,SAFIR] }\end{array}$ & $\begin{array}{l}\text { a) Observe proto-planetary } \\
\text { disks with the resolution } \\
\text { needed to detect Earths } \\
\text { in formation [FIRSI] } \\
\text { b) Trace the chemical } \\
\text { evolution of the early } \\
\text { Universe [Large } \\
\text { UV/Optical Imager] }\end{array}$ \\
\hline
\end{tabular}

TPF-C will directly detect Earth-like planets at visible wavelengths around nearby ( $\sim 30$ light-years) Sun-like stars. There are several driving requirements for this mission. The brightness ratio between the visible star and its reflected light from the planet is $10^{10}$, while the angle subtended between the star and Earth-like planet will be on the order of 100 milliarcseconds. The flux from an Earth-like planet is estimated to be roughly 0.05 photons $/ \mathrm{m}^{2} \mathrm{sec}$ over the full visible wavelength band from 0.4 to 0.9 microns. Therefore, a mirror of $\sim 25 \mathrm{~m}^{2}$, with a 5 nanometer rms surface, and with 20picometer wavefront control will be required to achieve a flux of about 1 photon/sec with sufficient contrast and signalto-noise ratio to actually "see the planet." Innovative processes to fabricate low-scatter surfaces and precision masks are some of the many advanced telescope technologies underway today. TPF-C will inherit a portion of the SIM optical bench and structures metrology that gives 200-picometer precision. A monolithic mirror is required because a segmented mirror's edge-scattering effects will degrade the contrast ratio. Improved detectors in the visible and, possibly, in the near-infrared are essential for spectroscopy of the detected objects.

TPF-I is presently planned to be carried out jointly with ESA as TPF-I/Darwin and will detect Earth-like planets around nearby Sun-like stars. This mission builds on SIM technologies that can provide the stable (nanometer) wavefronts needed for deep interferometric nulling at wavelengths of 6-17 microns. The current baseline configuration of this 
mission is 4-5 spacecraft, one combiner and 3-4 collectors with about 4-meter diameter apertures in a linear array, with a maximum separation of a few hundred meters. JWST technologies are basic to the optics, detectors, and cooling. TPF-I will require precision formation flying techniques so that the light from its independent telescopes can be combined and controlled to an accuracy of much less than one wavelength. In addition to requiring large aperture telescopes, TPF-I will need formation control algorithms, optical beam combiners, micro-Newton thrusters, laser metrology, and inter-satellite navigation.

Once the initial suite of planet finding missions, SIM, TPF-C and TPF-I/Darwin, has been completed, it is likely that an additional mission will be needed to make more detailed observations of suitable planetary atmospheres. The Life Finder (LF) might operate at either visible or infrared wavelengths depending on the scientific imperatives and technological capabilities available at the time. A factor of at least 10 increase in collecting aperture and thus in spectral resolution would allow searches for trace gasses such as $\mathrm{N}_{2} \mathrm{O}$ and $\mathrm{CH}_{4}$ that might elucidate the evolutionary state of habitable planets or reveal more about the metabolic processes of life-forms identified by the first missions.

\subsection{Role for smaller, competitively-selected missions and new technology}

The path towards characterizing planetary systems includes important roles for smaller, competitively selected missions. Technological breakthroughs already in sight are the lifeblood of this Roadmap, but not all can be predicted in advance. Some additional possibilities include improved methods for suppressing starlight in coronagraphs and nulling interferometers, improved concepts for operating SIM to improve its accuracy, or improved detector technology. Such improvements would enhance the performance of the planned mission sequence, or could be incorporated in competitively selected smaller missions, but do not change the basic scientific approach or requirements. The technical challenges facing TPF-C and TPF-I could be tested at reduced risk in the space environment with smaller scale, spaceborne missions: either an optical coronagraphic imager or an structurally connected infrared interferometer. Both have been discussed and/or proposed for \$350-450M Discovery line, either as US-only, or in conjunction with other countries. Emerging technologies to produce low-cost, rapid development large optics with controlled surfaces may enable a new class of affordable competed missions. A space telescope with an aperture of 2 meters, outfitted with adaptive optics and a coronagraph, could not detect Earths, but can detect analogs of Jupiter and Saturn orbiting between 5-20 AU from nearby stars. Similarly, a nulling interferometer operating at 3-5 microns over a 10-m baseline could study infrared emission from hot Jupiters. In both cases, a modest spectrometer could detect many of the molecular constituents of the atmospheres of giant planets, yielding insights about the chemical composition and origin of these planets. The light rejecting technologies by which these telescopes would block the starlight would be similar to those necessary for TPF-C or TPF-I, providing valuable technical insights about the more difficult goal of detecting Earths. A proof of concept for either approach might be feasible at the cost of a Discovery-Class mission.

\subsection{Research and Analysis}

Detecting and characterizing extrasolar terrestrial planets poses both considerable technological and scientific challenges. While substantial investments in large space-based telescopes will be required to make significant progress in this field, the ultimate scientific payoff from these missions will require not only the technological capabilities, but a strong scientific foundation from an active, interdisciplinary scientific community. Both precursor and supporting observations from space-based and ground-based telescopes, as well as a rich program of theoretical and interdisciplinary research will be needed. Theoretical, laboratory, and field research will provide end-to-end mission support by supplying crucial new ideas, context and information relevant to mission planning, design and science priorities, and by providing the expertise and tools to convert the hard-won spacecraft measurements into new scientific understanding. NASA will build and maintain this interdisciplinary science community via competed R\&A programs, of which the TPF Foundation Science program, NASA Astrobiology Institute, the Astrophysics Theory Program, the Origins of Solar Systems Program, the Astrophysical Data Program (ADP), and the Interdisciplinary Exploration Science program are current examples. Theoretical and multidisciplinary scientific research should also be integrated into the fundamental mission design to address scientific challenges that are critical to the mission's key goals.

\subsection{Unique Education and Public Engagement Opportunities}

The missions and programs that support the telescopic search for Earth-like planets and habitable environments around other stars present distinct opportunities to advance NASA's Strategic Objective \# 13: 
Use NASA Missions and other activities to inspire and motivate the Nation's students and teachers, to engage and educate the public, and to advance the scientific and technological capabilities of the

Nation.

This lofty goal serves down-to-Earth needs. We must generate and sustain an ample workforce of scientists and engineers - some of those who will implement the missions in this Roadmap are now only in elementary school. A strong education program provides 1) a necessary return on the public's investment in science exploration; 2) develops pathways for students to enter the fields of science, technology, engineering, and mathematics (STEM) careers, and 3) stimulates the public appetite for NASA Missions. Beyond the STEM careers themselves, the broader public must achieve a basic level of scientific literacy. This is good for the Nation and our democracy, and is a prerequisite for the support that a multi-decadal program of exploration and discovery requires.

NASA will need to plan proactively for the worldwide interest that the discovery of Earth-like worlds will cause. The quest has the potential to enter the mainstream of popular culture, capturing the public imagination at levels unmatched since the height of the Apollo program. Yet, the 30-year research timeline and attendant capital investment requires sustained public support. Unlike Apollo, this will be a journey, not a race. To support this major effort, the public needs to be emotionally engaged in the excitement of the missions, and to be rationally informed about the goals and accomplishments along the way.

\section{SUMMARY AND CONCLUSIONS}

There are few scientific questions that excite both the scientific community and the general public as much as the search for habitable planets and life beyond the solar system. The program described in the NASA Roadmap as summarized here will provide discoveries on short-, mid-, and long-term time scales, maintaining a sense of excitement to inspire a new generation of young people to take up scientific and technical careers and keep alive in society at large a sense of wonder about the universe. As only NASA can.

\section{ACKNOWLEDGEMENTS}

This work was carried out, in part, at the Jet Propulsion Laboratory, California Institute of Technology, under a contract with the National Aeronautics and Space Administration. 